\title{
Microwave time and frequency standards
}

\author{
Helmut Hellwig \\ National Bureau of Standards, Boulder, Colorado 80303
}

(Received February 1, 1979.)

\begin{abstract}
Today's time and frequency standards which are in active use ranging from the definition of the second to spacecraft applications are all based on atomic resonances in the microwave region controlling high-performance quartz crystal oscillators. The present status of these standards will be presented focusing in particular on cesium, hydrogen, and rubidium devices, as well as on new quartz crystal standards. A coherent picture of their physical principles and limitations is given based on the common attempt to reduce perturbations and Doppler effects. The sharpness of the resonance line is linked to the flicker noise stability limit of the various standards, and some speculation is made on where and how much further improvements may occur. It is demonstrated how new design concepts and new physical methods most likely will significantly improve the accuracy, stability, and practical usefulness of time and frequency standards.
\end{abstract}

\section{INTRODUCTION}

Today's precision clocks and frequency standards are, for the most part, based on quartz crystal oscillators and on atomic resonances. In particular, the resonances of cesium, rubidium, and hydrogen in the microwave region have found very wide laboratory use and, in addition, numerous technical applications. In fact, rubidium standards and cesium standards have been commercially available for 2 decades and have undergone numerous improve ments and refinements [McCoubrey, 1966; Hellwig. 1975]. The 1960's saw refinements in atomic clock design and their use in many scientific applications and in the generation of time on a worldwide basis. The 1970's can be characterized by the parallel events of fundamental, physical, and design breakthroughs in atomic as well as crystal standards and the beginnings of large-scale system use of precision oscillators in communication, navigation, and scientific studies. In this paper we first briefly look at this broadened spectrum of applications. Then we discuss the characterization of the performance of time and frequency standards including a summary of current performance, followed by an attempt at depicting the underlying physical principles and limitations regarding accuracy and stability. Finally, some speculation is made on where and how further improvements may occur.

\footnotetext{
This paper is not subject to U.S. copyright. Published in 1979 by the American Geophysical Union.
}

This last part includes a brief sketch of new design concepts and new physical methods.

\section{APPLICATIONS OF TIME AND FREQUENCY STANDARDS}

The application which has now become a classical use of precision time and frequency standards is the generation of time. This started with quartz crystal oscillators in the 1920's. These soon found service as workhorses in timekeeping and in interpolation between astronomical timefixes, while the primary frequency and time reference remained the rotating earth via astronomical observations. In the mid-1950's it was experimentally shown that cesium devices were usable as clocks for the actual generation of time and that cesium clocks significantly surpassed the performance of astronomical clocks [Essen and Parry, 1956]. As a consequence, new time scales called atomic time (AT or TA) were established to be maintained in parallel with the official astronomical time (family of universal times, UT) [Bulletin Horaire, 1965]. The increasing availability of commercial clocks since the late 1950's caused an increasing number of laboratories to generate atomic time scales. The demonstrated timekeeping uniformity exceeded that of astronomical times by orders of magnitude [Smith, 1972] . This led to the redefinition of the duration of the second in terms of the cesium resonance in 1967 [Seances, 1968]. In a further logical move the internationally coordinated time scales, internation- 
al atomic time (TAI) and coordinated universal time (UTC), were unified in 1972 [Smith, 1972]. TAI and UTC are the basis for official or legal time, which is made available by the responsible laboratories in the different countries. These local time scales, UTC $(i)$, are always an excellent approximation to UTC, normally differing from it by at most a few microseconds. We must note that for all practical uses the UTC $(i)$ signals are essential, since neither UTC nor TAI are available as physical signals; data are available via a report from the Bureau International de l'Heure after-the-fact in the form UTC-UTC $(i)$. We shall discuss only radio astronomy and relativity as two examples among the great many uses of precise time and frequency standards in science.

One application is radio astronomy, in particular, very long baseline interferometry (VLBI) [Klemperer, 1972]. In a typical VLBI experiment, two or more radio antennas receive signals from one or more stellar radio sources. The larger the distance between the locations of the radio antennas, the higher the resolution. However, it is essential to provide for coherence between these remote sites. The coherence can be provided by highly stable time bases located at each antenna site. These clocks provide, independently, phase coherence over observation times of sometimes many hours. The recorded signals then can be compared at any time after the actual observation; interference fringes are produced by comparison of the two or more recordings. Since the observations are done in the microwave region and observation times of many hours are common, clock stabilities of $10^{-14}$ or even better have been required in VLBI [Fanselow, 1977]. Of particular use in this regard have been hydrogen maser standards [Rogers et al., 1976].

An obvious major application in science is relativity. Fundamental experiments which observe changes in frequency and in accumulated time of clocks moving relative to each other and in different gravitational potentials have been made. Such experiments include clocks in airplanes [Haefele and Keating, 1972; Reisse, 1976; Williams, 1976; C. O. Alley and L. S. Cutler, private communication, 1976]. Proposals for spacecraft experiments have been made [Kleppner et al., 1970]. Of particular interest have been experiments of clock ensembles flown in commercial airliners around the world, demonstrating experimentally about a $400 \mathrm{~ns}$ time difference between clocks flown with the rotation of the earth compared with clocks flown against the rotation of the earth [Haefele and Keating, 1972]; also, a hydrogen maser space probe was sent in a 4-hour flight $10,000 \mathrm{~km}$ into space, returning in a ballistic trajectory back to earth [Vessot and Levine, 1977]. This latter experiment has provided an accuracy of $10^{-4}$ in the validity of the gravitational red shift.

In the area of technological applications we shall again confine ourselves to a few important examples. We shall ignore most of the large number of uses ranging from references and calibrations of almost any frequency- or time-based measurement to providing time for the operation of national power networks (permitting precise measurement of the phase and thus effective switching between different networks) to the control of television broadcasts (providing color purity via phase stability and switching of different broadcast generating stations into the major networks).

The most important use of time standards has always been navigation, that is, position finding with respect to stellar observations or man-made radio signals. In a more general sense, position finding amounts to obtaining a four-dimensional solution for the three spatial coordinates and the one time coordinate. A classical way of position finding on the surface of the earth involves the observation of star positions and the use of a table giving the evolution of their positions in time referenced to some fixed location on the earth. If such an observer is provided with a sufficiently precise clock, he can infer his own position on the surface of the earth.

A major space-based navigation system is presently being deployed in its first testing phase. It makes use of atomic clocks on board orbiting satellites [Butterfield, 1976]. Rubidium and cesium standards [McCaskill et al., 1978; Ringer et al., 1975] are currently in operation on board space vehicles, and improved rubidium or cesium standards, or even hydrogen devices, are envisioned for the final system. Position finding is fundamentally accomplished by obtaining range data for which, in principle, time measurements are converted to length measurements via the speed of light. Since a radio signal propagates at the speed of light, that is, approximately $3 \times 10^{8} \mathrm{~m} \mathrm{~s}^{-1}$, a measurement precision of $1 \mathrm{~ns}$ in time is equivalent to a distance or position accuracy of $0.3 \mathrm{~m}$. There- 
fore if the clocks on board the satellite are to provide a clock-limited position accuracy of better than 0.3 $\mathrm{m}$, a 1-ns operational time coherence of these clocks is needed. In other words, a clock performance of about $10^{-14}$ is required for time periods of 1 day; correspondingly better performance is needed if independent time coherence preservation over longer time periods is desired.

A user of this system can have an equivalently precise clock, in which case he would need only two satellites for position finding on the surface of the earth or three satellites for position fixes in three dimensions. If he can acquire signals from four satellites nearly simultaneously, he needs only to interpolate over the total required observation time which may not exceed several minutes and thus use an inferior clock. In this case he has to solve the total equation for the four coordinates, the three spatial ones and the time coordinate, in order to get a complete position fix from the system.

In order to make this global positioning system feasible, major clock development has recently taken place concentrating on all three classical systems, rubidium [Ringer et al., 1975], cesium [McCaskill et al., 1978; George and Vulcan, 1977], and hydrogen [Sabisky and Weakliem, 1978; Walls and Howe, 1978; Levine et al., 1978; Wang et al., 1977]. Significant improvements in clock performance as well as satisfactory clock performance and clock survival under the conditions of spacecraft launch and operation have been achieved. In addition, novel ideas are being incorporated into the time and frequency standard designs, which we will discuss later.

\section{CHARACTERIZATION AND PRESENT STATUS OF TIME AND FREQUENCY STANDARDS}

Time and frequency standards can be characterized in numerous ways. However, most frequently the so-called frequency and time domain stability characteristics are given. Frequency domain stability is typically expressed in terms of the one-sided spectral density of frequency fluctuation $S_{y}(f)$ or the one-sided spectral density of phase fluctuations $S_{\phi}(f)$, where $f$ is defined as the Fourier frequency offset from the carrier $\nu_{0}$ [Allan, 1966; Barnes et al., 1971; Cutler and Searle, 1966; Rutman, 1977]. These two quantities are related by (1):

$$
S_{y}(f)=\frac{f^{2}}{v_{\mathrm{o}}^{2}} S_{\phi}(f)
$$

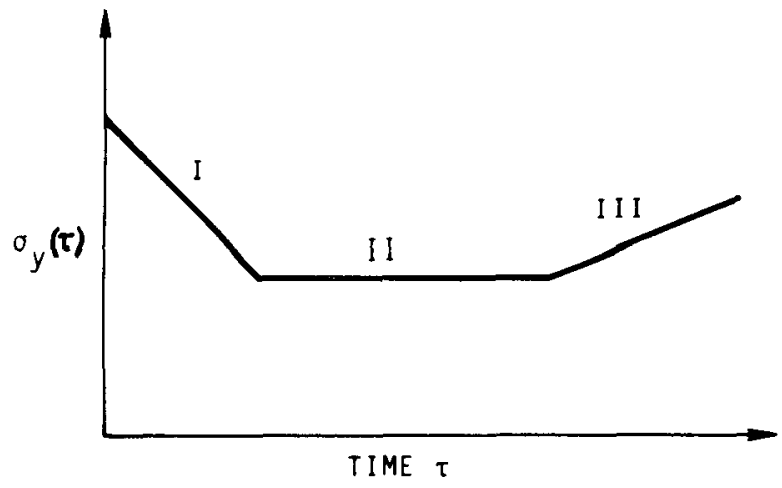

Fig. 1. Typical frequency stability behavior of a frequency standard. The two-sample variance is $\sigma_{y}^{2}(t)$.

In the time domain the typically used quantity is the two-sample (Allan) variance $\sigma_{y}^{2}(\tau)$ defined by [Allan, 1966; Barnes et al., 1971]:

$$
\sigma_{y}^{2}(\tau)=\left\langle\left(\bar{y}_{k+1}-\bar{y}_{k}\right)^{2} / 2\right\rangle
$$

where $\bar{y}$ is the fractional frequency fluctuation measured during a sample time $\tau$ and the brackets denote an infinite time average. In (2) it is assumed that there is no dead time between successive measurements. The bandwidth used in the measurement is an additional, implicit parameter [Allan, 1966; Barnes et al., 1971; Cutler and Searle, 1966] and should always be noted explicitly; in many cases it may significantly affect the actual value of $\sigma_{y}(\tau)$. Since interpretations of clock performance are most commonly related to $\sigma_{y}(\tau)$, we shall use it exclusively in the following discussions.

An idealized plot for all precise time and frequency standards is shown in Figure 1 [Hellwig, 1975]. The first part, I, of this stability plot can be characterized by the equation:

$$
\sigma_{y}(\tau)=K_{1} \tau^{\alpha_{1}}
$$

It is determined by the noise processes present in the standard. The second part, II, is called the flicker of frequency floor, which describes an independence of $\sigma_{y}(\tau)$ from the averaging time. We shall call this value $\sigma_{y F}$. The level of $\sigma_{y F}$ depends on the particular frequency standard and is not fully understood in its physical basis, but relates to fluctuations in the value of critical frequency-determining parameters of the standards. The last section, III, of the curve can again be characterized by 
TABLE 1. Typical performance data of commercially available standards in a benign field environment

\begin{tabular}{llcccc}
\hline & \multicolumn{1}{c}{$K_{1}$} & $\sigma_{y F}$ & $K_{2}$ & $D$ (per day) & Accuracy \\
\hline H (active) & $1 \times 10^{-12} \mathrm{~s}$ & $1 \times 10^{-14}$ & $3 \times 10^{-17} \sqrt{\mathrm{s}^{-1}}$ & $10^{-15}$ & $1 \times 10^{-12}$ \\
Cs & $5 \times 10^{-11} \sqrt{\mathrm{s}}$ & $1 \times 10^{-13}$ & $3 \times 10^{-17} \sqrt{\mathrm{s}^{-1}}$ & $10^{-15}$ & $2 \times 10^{-11}-7 \times 10^{-12}$ \\
& $7 \times 10^{-12} \sqrt{\mathrm{s}}$ & $5 \times 10^{-14}$ & $3 \times 10^{-17} \sqrt{\mathrm{s}^{-1}}$ & $10^{-15}-10^{-14}$ & $7 \times 10^{-12}$ \\
$\mathrm{Rb}$ & $5 \times 10^{-12} \sqrt{\mathrm{s}}$ & $5 \times 10^{-13}$ & $3 \times 10^{-15} \sqrt{\mathrm{s}}$ & $1 \times 10^{-12}$ & $1 \times 10^{-9}$ \\
X-tal & $1 \times 10^{-12} \mathrm{~s}$ & $5 \times 10^{-13}$ & $3 \times 10^{-15} \sqrt{\mathrm{s}}$ & $1 \times 10^{-11}$ & $\cdots$ \\
\hline
\end{tabular}

( $\mathrm{H}$ standards are not commercially available: data are from designs and uses approximating those of the other standards).

$$
\sigma_{y}(\tau)=K_{2} \tau^{\alpha_{2}}
$$

The coefficients $K_{2}$ and $\alpha_{2}$ cannot usually be determined very accurately because of the long measurement times needed in order to obtain statistical confidence. Also, these coefficients are often subject to environmental influences and are thus not stable. (The sharp corners between parts I and II and II and III are not actually observed. It is a useful simplification for discussion purposes.)

A very important term which should be subtracted from the data before a characterization of the standard, such as Figure 1 , is made is linear frequency drift $D$. If linear frequency drift is present and not removed from the data, it would appear in Figure 1 as $\alpha_{2}=+1$. Since linear frequency drift signifies systematic changes with time of frequency-determining parameters, a statistical description by $\sigma_{y}(\tau)$ is an inefficient method. The effect on the time-keeping ability of the standard if $D$ is not known is highly important. Equation (5) shows the time error $T$ at the elapsed time $l$ after synchronization [Hellwig et al., 1975b]:

$$
T(t)=T_{0}+R_{0} t+\frac{1}{2} D t^{2}+\cdots \varepsilon(t)
$$

We see that if $D \neq 0$, a time error accumulates quadratically with elapsed time. $R_{0}$ is the rate offset (fractional frequency difference) between the measured clock and an (ideal) reference clock, and $\varepsilon(t)$ contains all other frequency fluctuations as described by Figure 1 and (1)-(4). The importance of $D$ can be illustrated by assuming that there is an uncorrected drift in a clock of $D=2 \times 10^{-10}$ per day corresponding to a good crystal oscillator. This drift would accumulate over 10 days a time error of approximately $1 \mathrm{~ms}$.

Using the coefficients of (3)-(5) and $\sigma_{y F}$, we shall characterize the presently available precise time and frequency standards in Tables 1 and 2 and the corresponding Figures 2, 3, and 4. In Table 1 and Figure 2 we depict the performance which has been measured using commercial devices in actual application conditions [Hewlett-Packard, Frequency and Time Systems, Frequency Electronics, Efratom, private communications and catalog information; Vessot et al., 1977; Reinhardt et al., 1976; Rogers et al., 1976]. All devices referred to are commercially available with the exception of hydrogen, where we used data from devices con-

TABLE 2. Best measured performance data in a controlled laboratory environment of commercial (com) and laboratory (lab)

\begin{tabular}{|c|c|c|c|c|c|}
\hline & $\boldsymbol{K}_{1}$ & $\sigma_{y F}$ & $K_{2}$ & $D$ (per day) & Accuracy \\
\hline $\mathrm{H}$ active (lab) & $5 \times 10^{-13} \mathrm{~s}$ & $<7 \times 10^{-16 *}$ & $1 \times 10^{-17} \sqrt{\mathrm{s}}^{-1}$ & $\left(<10^{-15}\right)$ & $1 \times 10^{-12}$ \\
\hline H passive (lab) & $1 \times 10^{-12} \sqrt{s}$ & $<3 \times 10^{-15_{*}}$ & $\left(<1 \times 10^{-17} \sqrt{\mathrm{s}^{-1}}\right)$ & $\left(<10^{-15}\right)$ & $1 \times 10^{-12}$ \\
\hline Cs standard (com) & $3 \times 10^{-11} \sqrt{s}$ & $5 \times 10^{-14}$ & $(0 ?)$ & $\approx 10^{-15}$ & $5 \times 10^{-12}$ \\
\hline Cs high perf (com) & $7 \times 10^{-12} \sqrt{\mathrm{s}}$ & $1 \times 10^{-14}$ & $(0 ?)$ & $10^{-15}-10^{-14}$ & $2 \times 10^{-12}$ \\
\hline Cs laboratory & $1 \times 10^{-12} \sqrt{s}$ & $7 \times 10^{-15}$ & (0?) & $<10^{-15}$ & $9 \times 10^{-14}$ \\
\hline $\mathrm{Rb}$ (com) & $2 \times 10^{-12} \sqrt{s}$ & $1 \times 10^{-13}$ & $1 \times 10^{-15} \sqrt{s}^{-1}$ & $1 \times 10^{-13}$ & $\left(1 \times 10^{-10}\right)$ \\
\hline X-tal oscillator (com) & $2 \times 10^{-13} \mathrm{~s}$ & $2 \times 10^{-13}$ & $\left(3 \times 10^{-15}{\sqrt{s^{-1}}}^{-1}\right.$ & $1 \times 10^{-12}$ & $\cdots$ \\
\hline dual X-tal (lab) & $2 \times 10^{-12} \mathrm{~s}$ & $6 \times 10^{-14}$ & $\left(3 \times 10^{-15} \sqrt{\mathrm{s}^{-1}}\right)$ & no data & $\cdots$ \\
\hline
\end{tabular}
standards

Data in parenthesis are not directly measured but inferred.

*These values, as well as the corresponding portions of the curves in Figure 4, are not confirmed 'flicker floors' but upper limits; any real flicker floor, if present, is masked by the I and III portions of the instability behavior (compare Figures 1 and 4). 


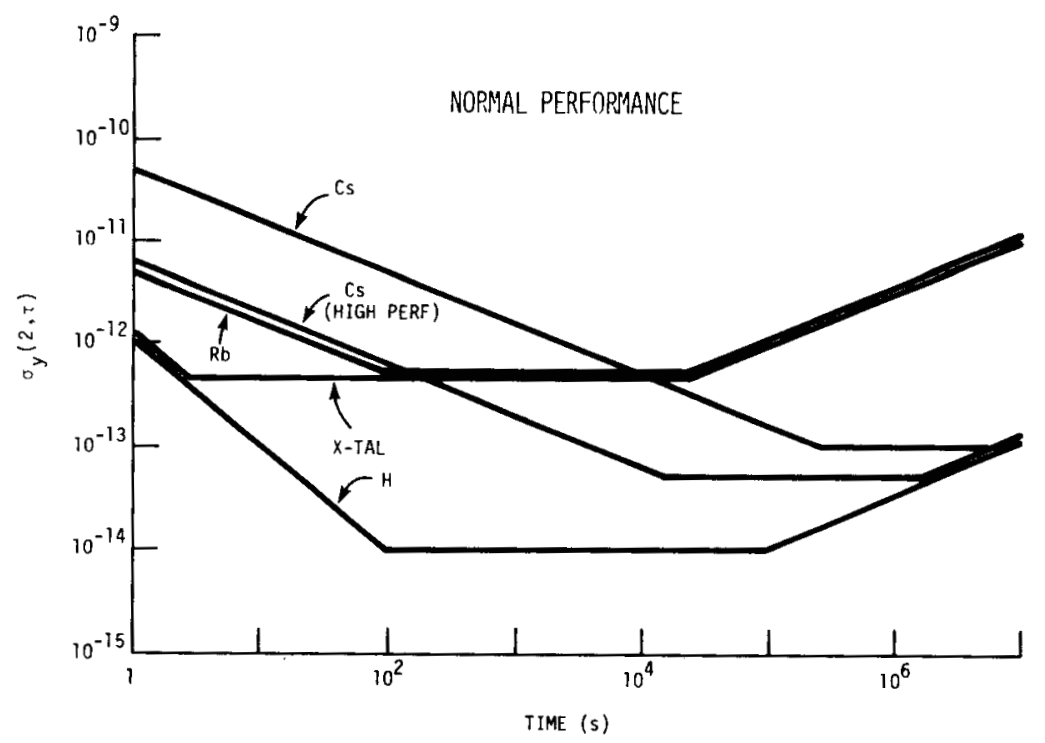

Fig. 2. Typical frequency stability of frequency standards of a commercial type.

structed by the Smithsonian Astrophysical Observatory and NASA.

In Table 2 and Figures 3 and 4 we list data on classes of standards, i.e., on quartz crystal, rubidium, cesium, and hydrogen standards which are based on the best published data experimentally obtained with such standards [Levine et al, 1978; Walls and Howe, 1978; Mungall, 1978; Percival, 1976; Becker, 1976, 1977; Wineland et al., 1976;
Hellwig, 1975; Stein et al., 1978; NBS experiment] These data are usually measured under very benign laboratory conditions, which typically include temperature variations in the environment of the standards of less than $1^{\circ}$, magnetic field stability to better than a tenth of a percent, and no vibration or acceleration, etc. In a later table we list for comparison what we believe can be ultimately achieved with these and other devices.

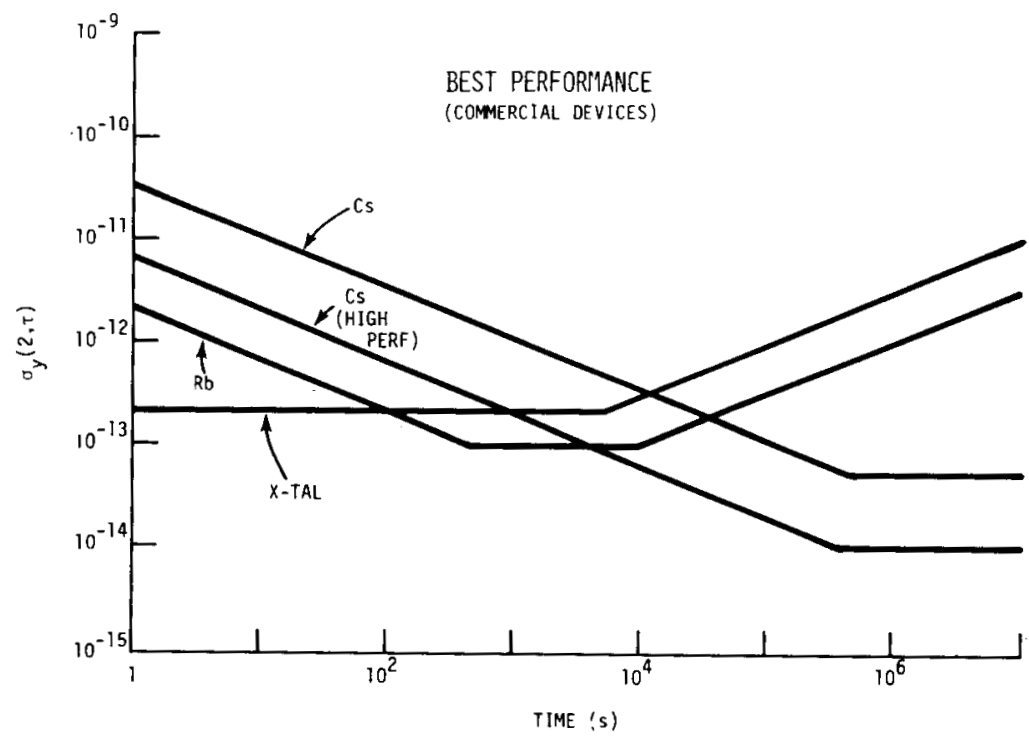

Fig. 3. Best measured frequency stability of commercially available frequency standards. 


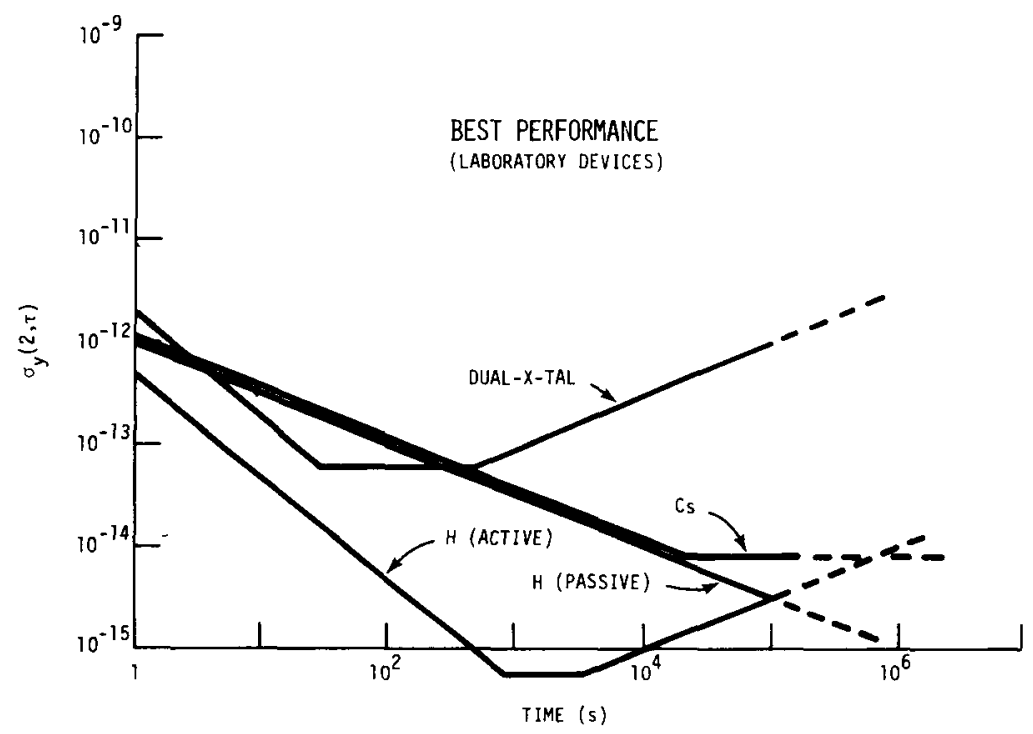

Fig. 4. Best measured frequency stability of laboratory-type frequency standards.

\section{PHYSICAL PRINCIPLES AND LIMITATIONS OF FREQUENCY STANDARDS}

Frequency standard concepts and designs are based on the following five principles: (1) minimized first-order Doppler shifts and line broadening, (2) reduced second-order Doppler effects, (3) freedom from perturbations, (4) as high a line- $Q$ as possible, (5) as high a signal-to-noise ratio as possible. These five principles are not independent of each other. For example, the ideal situation may appear to be a single atom at rest in free space observed for an infinite length of time. This would fulfill four of the above requirements, but the signal-to-noise ratio would be zero. This brings us to an important observation: accuracy and lack of biases are not useful in many cases if measurement takes an excessive amount of time. The maximum reasonable sampling time per measurement of one parameter may be taken as $10^{4} \mathrm{~s}$ in order to achieve a certain desired accuracy $\sigma_{A}$ with adequate confidence. This desire leads to some limits on the coefficient $K_{1}$ of (3). For noise processes described by $\alpha_{1}=-\frac{1}{2}$, one wishes to have $K_{1} \leq \sigma_{A} 10^{2} \mathrm{~s}^{1 / 2}$, and for $\alpha_{1}$ $=-1, K_{1} \leq \sigma_{A} 10^{4} \mathrm{~s}$. For example, if a beam frequency standard development targets $10^{-15}$ as the ultimate accuracy, a 1-s stability performance of $10^{-13}$ is desirable. (In clock operation over very long time periods (weeks to years) these 'requirements' on $K$ can be relaxed for cases such as time keeping, where a continual improvement of the stability with averaging time is a main objective and flicker or walk of frequency are not limiting.)

Equation (6) gives the approximate relationship between two of the above parameters and the noise-limited (short term) stability for the case of a frequency lock loop (white noise of frequency):

$$
\sigma_{\nu}(\tau) \simeq 1 /[Q S / N(\tau)]
$$

$S / N(\tau)$ is the signal-to-noise ratio which, of course, is a function of the sampling time $\tau$. For each particular design of a frequency standard there seems to be a limit in the product $Q \cdot S / N$. For example, in quartz crystal standards, $S / N$ is given by the interrogating or driving microwave power level. If certain power levels are exceeded (of the order of $10-100 \mu \mathrm{W}$ ), then the amplitude of crystal oscillations begins to be nonlinear, and other modes are excited, leading to a deterioration of $Q$ and thus to an ultimate reduction of the stability. In another example the $S / N$ of a hydrogen maser can be increased by increasing the intensity of the hydrogen beam, thus achieving the storage of more and more particles in the storage bulb. If certain hydrogen densities are exceeded, line broadening, i.e., $Q$ deterioration, occurs owing to spin exchange collisions between the hydrogen atoms. This effect appears to limit traditional hydrogen masers to $K$, $=10^{-13} \mathrm{~s}$. 
Equations (7) and (8) give the relationship between $S / N$ and the physical parameters of atomic standards which, typically, are shot noise limited ( $n$ is the number of signal events per unit time) and mechanical oscillators such as crystals and superconducting cavities ( $P$ is the driving power, $k T$ is the thermal noise):

$$
\begin{gathered}
S / N=\sqrt{n \tau} \\
S / N=\sqrt{P \tau / k T}
\end{gathered}
$$

The design of all atomic standards is dictated by an attempt to minimize first-order Doppler frequency shifts and line broadening. There are two fundamental ways to go about this. (1) The atoms can be confined in a region less than $\lambda / 2$, where $\lambda$ is the wavelength of the interrogating radiation. This is the case in the hydrogen storage principle and the rubidium gas cell. (2) The propagation direction of the interrogating radiation (propagating at approximately the speed of light) is arranged perpendicular to the movement of the atoms. Then a reduction is achieved to the degree that perpendicularity can be realized and net traveling waves in the direction of the atomic motion can be avoided. For example, a beam divergence of $10^{-3}$ rad going through a cavity with $Q=1000$ (equivalent to an energy in the traveling wave of $10^{-3}$ of the stored energy) with the Poynting vector $10^{-4}$ rad away from perpendicularity will show a residual Doppler broadening of about $10^{-6} W_{D}$ and a frequency shift of $10^{-7} v_{p} / c_{p}$, where $W_{D}$ is the width at half intensity of a normally Doppler-broadened line for a Maxwellian velocity distribution as given by (9), $v_{p}$ is the most probable atomic velocity, and $c_{p}$ is the velocity of the electromagnetic radiation:

$$
W_{D}=2(\ln 2)^{1 / 2} \nu_{\mathrm{o}}\left(v_{p} / c_{p}\right)
$$

The above quoted numerical values are close to being typical for a beam type standard such as cesium using $v_{p}=100 \mathrm{~m} / \mathrm{s}$. We see that the Doppler line broadening is completely negligible, whereas the Doppler frequency shift bias becomes nonnegligible at the $10^{-13}$ level.

The second-order Doppler shift is given by (10):

$$
\Delta v / v=\frac{1}{2}(v / c)^{2}
$$

Since this is a fundamental shift originating from relativity and relating to time dilation between a moving object (atoms) and an observer at rest (cavity), no particular design can affect this shift except an actual reduction in the atomic velocity.

Freedom from perturbation is a fundamental problem in all frequency standards. In atomic frequency standards it is the deviation from the ideal of an atom at rest in free space. The effects of fields such as magnetic fields is usually not a fundamental limitation, since they (1) can be controlled by present day technology and (2) can be measured using the Zeeman transitions in the atom itself as a highly sensitive magnetometer [Kleppner et al., 1965; Glaze et al., 1977]. More serious perturbations are those which conflict with the idea of obtaining a good stability, that is, a high line- $Q$ and a high signal-to-noise ratio. Good stability usually requires that a sufficiently large number of atoms be observed (see equation (7)); however, as was mentioned above, these atoms ultimately begin to collide with each other and thus produce increased relaxation effects together with frequency shifts. Beam tubes such as cesium suffer least from these limitations; in fact, present day cesium devices use beam intensities which are still far removed from these limitations. Other methods of confinement, such as those in hydrogen masers where physical walls are used or in rubidium where a buffer gas is used, suffer unavoidably from the collisions which must occur in this design.

This brings us to the question of line- $Q$. Equation (11) describes the relationship between line- $Q$ and observation time, where $\tau_{c}$ is the coherent observation time, that is, the time characterizing the relaxation process of the oscillating medium:

$$
Q_{l}=r \nu_{0} \tau_{c}
$$

The coefficient $r$ in (11) depends on the particular physical relaxation process. However, $r$ is of the order of $\pi$ for most practical purposes. We see from (11) that the longer the coherent observation time, the higher the line- $Q$. The desire to make $\tau_{c}$ as large as possible conflicts with the ideal of freedom from perturbation and high signal-to-noise ratio. Very long observation times decrease the available signal-to-noise ratio if the same freedom from perturbation as in shorter observation times is to be achieved, or signal-to-noise ratio can be maintained while increasing the perturbation. For example, in cesium the attempt to increase $\tau_{c}$ leads to a longer beam tube with an attendant reduction of usable beam divergence angle and thus reduced signal-to-noise ratio. In addition, there are obvious limitations to the practicality of this approach, 
including environmental problems such as uniform and stable magnetic fields over very long regions of space.

For quartz crystal resonators there is a substantial list of perturbations which, of course, relate to the problem of frequency drift. Major perturbations in crystal resonators are believed to be due to crystal lattice imperfections. The plating of metallic electrodes directly on the crystal surface and the crystal mounting supports lead to mass loading and induced mechanical stress. Time changes of these parameters and diffusion of metal into the crystal lattice cause frequency fluctuations and drift. In addition, time varying temperature gradients may be present. The environment of the quartz crystal resonator, i.e., the always imperfect vacuum within the enclosure, may be of importance but presently appears to be under better control than the other parameters.

We shall now entertain some speculation on the causes for the flicker of frequency stability limitation which is displayed universally by all frequency standards. We assert that the flicker of frequency noise floor is related to the resonance line- $Q$ of the frequency standard under consideration, i.e., we can describe $\sigma_{y F}$ by the following simple relationship:

$$
\sigma_{y F}=F / Q_{l}
$$

where $F$ is a constant describing the particular properties of a class of standards. Equation (12) is supported by the fact that in most frequency standards the significant sensitivities to internal or external parameter changes of the standard are scaled by $Q_{i}$. In the following we list two examples:

1. Cesium beam standard. The most significant frequency bias is cavity phase shift [Becker, 1976; Mungall, 1978; Wineland et al., 1977; Hellwig et al., 1975a] Owing to a difference in cavity phase between the two ends of the cavity, as well as to a variation of cavity phase across the beam cross section (distributed cavity phase shift) [Jarvis, 1975; Garvey et al., 1978; Allan et al., 1977; Hellwig et al., 1975a; Becker, 1978], frequency shifts can be approximated by

$$
\Delta v / \nu \simeq \delta / Q,
$$

where $\delta$ is a cavity phase variation.

2. Hydrogen. The most significant effect on hydrogen is cavity pulling due to a frequency offset or change $\Delta v_{c}$ in the cavity resonance frequency. This is approximately given by [Kleppner et al., 1965]
TABLE 3. Best measured data of commercial (com) and laboratory (lab) standards

\begin{tabular}{lccc}
\hline & $Q$ & $\sigma_{y F}$ & $F$ \\
\hline H (lab) (active) & $3 \times 10^{9}$ & $7 \times 10^{-16 *}$ & $2 \times 10^{-6}$ \\
Cs (lab) & $3 \times 10^{8}$ & $7 \times 10^{-15}$ & $2 \times 10^{-6}$ \\
Cs (com) & $4 \times 10^{7}$ & $1 \times 10^{-14}$ & $4 \times 10^{-7}$ \\
& $1 \times 10^{7}$ & $5 \times 10^{-14}$ & $5 \times 10^{-7}$ \\
Rb (com) & $2 \times 10^{7}$ & $1 \times 10^{-13}$ & $2 \times 10^{-6}$ \\
NH (gas cell) (lab) & $1 \times 10^{5}$ & $1 \times 10^{-10}$ & $1 \times 10^{-5}$ \\
X-tal (dual X-tal, lab) & $2 \times 10^{6}$ & $6 \times 10^{-14}$ & $1 \times 10^{-7}$ \\
Supercond cav (lab) & $10^{11}$ & $4 \times 10^{-16}$ & $4 \times 10^{-5}$
\end{tabular}

$F=Q \cdot \sigma_{y e}$

*This value is only an upper limit (compare Table 2).

$$
\Delta v / v \simeq\left(Q_{c} \Delta v_{c} / \nu_{\mathrm{o}}\right) / Q_{l}
$$

where $Q_{c}$ is the $Q$ value of the cavity.

In Table 3 we list the $Q$ values and the best estimates of $\sigma_{y}(\tau)$ values to date of a variety of standards [Glaze et al., 1977; Levine et al., 1978; Percival, 1976; Stein, 1975; Hellwig, 1975; Stein et al., 1978; Wineland et al., 1977]. The last column in Table 3 gives the resultant $F$ value following (12). It is interesting that the $F$ values cluster around $10^{-6}$. This may be just a chance coincidence, largely reflecting competitive levels of the designers' ingenuity; however, it is nevertheless very suggestive of some underlying, more fundamental, possibly generic physical limitation.

\section{FUTURE DEVELOPMENTS}

Parallel with the technology improvements and demands of the last few years, a resurgence of fundamental studies of accuracy and stability limitations in time and frequency standards has occurred. As a result, we can now make a better assessment and more accurate predictions of further performance improvements than has been possible in the past.

1. Cesium. The major accuracy limitation in cesium is probably related to the cavity phase shift and, in particular, to the distributed cavity phase shift. The latter effect is due to a nonuniform distribution of the phase of the interrogating electromagnetic radiation across the beam [Hellwig et al., 1975a, Jarvis, 1975]. As a result, different trajectories within the beam have different resonance frequencies, and the device resonance frequency depends on the contribution from and summation over the individual trajectories [Allan et al., 1977]. The distributed cavity phase shift therefore influences the frequency stability of the device via any effect which changes the distribution 
of trajectories of contributing atoms in the cavity. Since all of today's cesium beam tubes feature velocity dispersive state selection, trajectory changes can occur with microwave power level changes [Glaze et al., 1977], magnetic field changes (Majorana transitions) [Allan et al., 1977], as well as physical changes in oven and detector position and brightness changes across the oven output and the detector input surface [recent NBS hypothesis] Such effects at the detector may relate to electric potential changes affecting ion collection at the hot wire [Allan et al., 1977; Becker, 1978]. In addition, cesium standards may be stability limited owing to magnetic field effects, which include fluctuations in the average value of the magnetic field as well as in the distribution of the field along and across the beam. Another limitation might be pulling by the tails of the $m_{F} \neq 0$ transitions, whose intensities may change with microwave power level, trajectory location, etc. (see distributed cavity phase shift). Various attacks on these problems are possible, and some progress has been made. This includes the two-cavity/two-frequency method, which eliminates cavity phase shift [Jarvis et al., 1977; Garvey et al., 1978]; optical pumping, which greatly reduces velocity dispersion in state selection [Picque, 1977]; superconducting cavities, which reduce cavity phase shifts [Wineland, 1977]; narrow beams [Hellwig et al., 1975a]; velocity and magnetic field stabilizing servos [Hellwig et al., 1975a; Allan et al., 1977]. etc.

2. Hydrogen. The most fundamental accuracy limitation in hydrogen is the wall collision effect, and the principal limitations of stability relate to cavity pulling [Kleppner et al., 1965; Hellwig et $a l ., 1970]$. In addition to mechanically and thermally stabilizing the cavity resonance frequency a method has been demonstrated by which, at some sacrifice in short-term noise performance, the hydrogen resonance is interrogated passively, using two wellseparated modulation frequencies. The resultant signals are used to lock a crystal oscillator and, independently, the microwave cavity to the hydrogen resonance [Walls and Howe, 1978]. Several ideas on significantly reducing the size of the $1.4-\mathrm{GHz}$ resonant cavity, ranging from dielectric loading to radically different mode structures [Mattison et al., 1976; Peters, 1978], have been proposed and tested. For both active and passive hydrogen standards this may lead to significant size reductions at little or no sacrifice in performance. Motional frequency shifts which depend on mag- netic field inhomogeneities in the storage region of hydrogen appear [Crampton et al., 1977] avoidable by proper design [Walls and Howe, 1978]. The problem of hydrogen wall shift remains open for a solution [Vanier and Larouche, 1978]. It needs to be studied, taking advantage of the greatly increased experimental opportunities offered by the new device developments.

3. Rubidium. The principal limitation in the performance of rubidium relates to the use of buffer gas, limiting both accuracy and stability via a collisional frequency bias of the order of $10^{-9}$ [Davidovits and Novick, 1966; Missout and Vanier, 1975a] and via the fact that the buffer gas confines the radiating rubidium atoms to very small regions of space, thus causing an inhomogeneous line broadening. As a result, frequency shifts and instabilities occur owing to intensity and spectral gradients of the pumping light across the cavity, magnetic field inhomogeneities across the cavity, and the necessarily nonuniform distribution of the microwave interrogating radiation [Missout and Vanier, 1975b; Risley and Busca, 1978]. In simple words, the atom in the left part of the cavity experiences a different physical environment than an atom in the right part of the cavity. The fundamental solution to this problem is to allow individual rubidium atoms to sufficiently average, in a homogeneous way, the volume of the cavity and, if possible, to physically separate the process of optical pumping from the microwave interrogation. The former may be possible via significant reductions in the buffer gas densities combined with suitable wall coatings (J. Vanier, private communication, 1978). The latter may be achieved by a separation in space or in time (pulsing) of the optical pumping from the microwave interrogation processes [Risley and Busca, 1978] .

4. Crystal Oscillators. The general aim of improvement in crystal resonator design and performance rests with methods for eliminating effects of stress, mass loading, and impurity diffusion due to the electrodes, the surrounding gas, and the mounting of the crystal. The successes achieved with new doubly rotated crystal cuts [Kusters et al., 1977; Ballato et al., 1977] which apparently reduce stress effects (temperature gradients and acceleration/vibration sensitivity) by an order of magnitude, as well as the successful development of electrodeless specially mounted quartz crystal resonators [Besson, 1977], have reopened the field of crystal resonator design. The combination of 
these new resonators with either traditional circuits featuring low-noise and low-drift electronic components or with new circuits such as the dual-crystal standard [Stein et al., 1978] has led already to the results quoted in Table 2 . In the dual-crystal standard an active oscillator is locked to a passively interrogated crystal resonator much as in an atomic frequency standard. Further significant improvements, in particular in the flicker floor and the drift performance, can be expected rivaling the results presently obtained only with atomic frequency standards.

5. Cooling. Finally, we shall briefly discuss a more fundamental attack on most of the above discussed problems and limitations which, we believe, will have a tremendous effect on our fundamental laboratory and metrology capabilities. With presently available technology, especially with regard to the use of laser radiation pressure, cooling would have no significant role in the design of standards for practical field applications. However, more practical cooling techniques may become available in the future.

The parameter which appears either explicitly or hidden in (9)-(14) is the velocity. Reducing the velocity reduces the second-order Doppler effect in a fundamental way and increases the line- $Q$, thus decreasing practically all accuracy and stability limitations. It is known that cooled crystal resonators reach $Q$ values of above $10^{9}$ [Smagin, 1974] . The hydrogen maser will likely increase in line- $Q$ and decrease in the wall collision bias by cooling the cavity and bulb [Vessot et al., 1977]. Cooling of atoms (ions) has been experimentally demonstrated [Wineland et al., 1978], and even cooling of neutral beams [Ashkin, 1978; Bjorkholm et al., 1978; Hansch and Schawlow, 1975; Lethokov et al., 1977] such as cesium now seems feasible.

6. Summary. Table 4 summarizes our projections for the important parameters $Q_{l}, K_{1}$, and $\sigma_{y F}$ based on the opportunities discussed in para- graphs 1-4 above and, in addition, based on the assumption of cooling to about $3 \mathrm{~K}$. We see that one might expect to increase line- $Q$ values by an order of magnitude through cooling. This would result in flicker noise floors $\sigma_{y F}$ pushed lower by also an order of magnitude if our speculation of Table 3 and (12) has any validity. At normal operating temperatures the improvements in line- $Q$ are only small, if there are any, but we expect substantial improvements in short-term stability $\left(K_{1}\right)$, noise floor $\left(\sigma_{y F}\right)$, and accuracy due to novel design principles as outlined above.

Acknowledgments. The author is deeply indebted to all his colleagues working on time frequency metrology at the National Bureau of Standards. In particular, discussions and contributions by R. M. Garvey, S. R. Stein, F. L. Walls, and D. J. Wineland helped shape this paper.

\section{REFERENCES}

Allan, D. W. (1966), Statistics of atomic frequency standards, Proc. IEEE, 54, 221-230.

Allan, D. W., H. Hellwig, S. Jarvis, Jr., D. A. Howe, and R. M. Garvey (1977), Some causes and cures of frequency instabilities in cesium beam frequency stardards, Proceedings of the 31st Annual Frequency Control Symposium, pp. 555-561, Electronic Industries Association, Washington, D.C.

Ashkin, A. (1978), Trapping of atoms by resonance radiation pressure, Phys. Rev. Lett., 40, 729-732.

Ballato, A., T. Lukaszek, and E. P. EerNisse (1977), The force-frequency effect in doubly rotated quartz resonators, Proceedings of the 31st Annual Frequency Control Symposium, pp. 8-16, Electronic Industries Association, Washington, D.C.

Barnes, J., A. R. Chi, L. S. Cutler, D. J. Healey, D. B. Leeson, T. E. McGunigal, J. A. Mullen, W. L. Smith, R. Sydnor, R. F. C. Vessot, and G. M. R. Winkler (1971), Characterization of frequency stability, IEEE Trans. Instrum. Meas., IM-20, 105-120.

Becker, G. (1976), Recent progress in primary Cs beam frequency standards at the PTB, IEEE Trans. Instrum. Meas., IM-25, 458-465.

Becker, G. (1977), Performance of the primary Cs-standard of the PTB, Metrologia, 13, 99-104.

Becker, G. (1978), Research on Cs-beam frequency standard at the PTB: Beam optics Majorana transitions, IEEE Trans.

TABLE 4. Summary of projections for important parameters

\begin{tabular}{|c|c|c|c|c|c|c|c|}
\hline & \multicolumn{4}{|c|}{ Normal } & \multicolumn{3}{|c|}{ Cooled $(\simeq 3 \mathrm{~K})$} \\
\hline & $Q$ & $K_{1}$ & $\sigma_{y} r$ & Accuracy & $Q$ & $\sigma_{y F}$ & Accuracy \\
\hline $\mathbf{H}$ & $10^{10}$ & $k \times 10^{-13} \mathrm{~s}$ & $10^{-16}$ & $10^{-13}$ & $10^{11}$ & $10^{-17}$ & $10^{-14}$ \\
\hline Cs & $3 \times 10^{8}$ & $1 \times 10^{-13} \sqrt{s}$ & $10^{-15}$ & $10^{-14}$ & $3 \times 10^{9}$ & $10^{-16}$ & $10^{-15}$ \\
\hline $\mathbf{R b}$ & $10^{8}$ & $1 \times 10^{-13} \sqrt{\mathrm{s}}$ & $10^{-14}$ & $10^{-12}$ & \multicolumn{3}{|c|}{ no serious proposal yet for cooling } \\
\hline $\mathrm{X}$-tal & $4 \times 10^{6}$ & $1 \times 10^{-15} \mathrm{~s}$ & $10^{-14}$ & $\ldots$ & $10^{9}-10^{10}$ & $\cdots$ & \\
\hline
\end{tabular}

Projected data are highly speculative, possibly controversial, and should be viewed as rough indications only. 
Instrum. Meas., IM-27, 319.

Besson, R. J. (1977), A new electrodeless resonator design, Proceedings of the 31st Annual Frequency Control Symposium, pp. 147-152, Electronic Industries Association, Washington, D.C.

Bjorkholm, J. E., R. R. Freeman, A. Ashkin, and D. B. Pearson (1978), Observation of dipole forces exerted on atoms by intensity gradients of resonant light, Phys. Rev. Lett., 4I, 1361-1363.

Bulletin Horaire (1965), series J, no. 7, p. 2, Bureau Internationale de l'Heure, Paris, France.

Butterfield, F. E. (1976), Frequency control and time information in the Navstar/Global positioning system, Proceedings of the 30th Annual Frequency Control Symposium, pp. 371-374, Electronic Industries Association, Washington, D.C.

Crampton, S. B., E. C. Fleri, and H. T. M. Wang (1977), Effects of atomic resonance broadening mechanism on atomic hydrogen maser long-term frequency stability, Metrologia, 13, 131-136.

Cutler, L. S., and C. L. Searle (1966), Some aspects of the theory and measurement of frequency fluctuations in frequency standards, Proc. IEEE, 54, 136-154.

Davidovits, P., and R. Novick (1966), The optically pumped rubidium maser, Proc. IEEE, 54, 155-169.

Essen, L., and J. V. L. Parry (1956), Atomic and astronomical time, Nature, 177, 744-745.

Fanselow, J. L. (1977), VLBI and its current applications within the solar system, Proceedings of the 9th Precise Time and Time Interval Planning Meeting, pp. 85-96, Technical Information and Administrative Support Division, Goddard Space Flight Center, Greenbelt, Md.

Garvey, R. M., H. Hellwig, S. Jarvis, Jr., and D. J. Wineland (1978), Two-frequency separated oscillating fields technique for atomic and molecular beam spectroscopy, IEEE Trans. Instrum. Meas., IM-27, 349.

George, J., and A. I. Vulcan (1977), Development of a cesium beam clock for satellite application, Proceedings of the 31st Annual Frequency Control Symposium, pp. 542-550, Electronic Industries Association, Washington, D.C.

Glaze, D. J., H. Hellwig, D. W. Allan, and S. Jarvis, Jr. (1977), NBS-4 and NBS-6: The NBS primary frequency standards, Metrologia, 13, 17-28.

Haefele, J. C., and R. E. Keating (1972), Around the world atomic clocks: Predicted and observed relativistic time gains, Science, 177, 166-170.

Hänsch, T. W., and A. L. Schawlow (1975), Cooling of gases by laser radiation, Opt.Commun., 13, 68-69.

Hellwig, H. (1975), Atomic frequency standards: A survey, Proc. IEEE, 63, 212-229.

Hellwig, H., R. F. C. Vessot, M. W. Levine, P. W. Zitzewitz, D. W. Allan, and D. J. Glaze (1970), Measurement of the unperturbed hydrogen hyperfine transition frequency, $I E E E$ Trans. Instrum. Meas., IM-19, 200-209.

Hellwig, H., D. W. Allan, S. Jarvis, Jr., D. J. Glaze (1975a), The realization of the second, in Atomic Masses and Fundamental Constants, edited by J. H. Sanders and A. H. Wapstra, pp. 330-336, Plenum, New York.

Hellwig, H., D. W. Allan, and F. L. Walls (1975b), Time and frequency, in Atomic Masses and Fundamental Constants, edited by J. H. Sanders and A. H. Wapstra, pp. 305-311, Plenum, New York.
Jarvis, S., Jr. (1975), Molecular beam tube frequency biases due to distributed cavity phase variations, Nat. Bur. Stand. Tech. Note, 660.

Jarvis, S., Jr., D. J. Wineland, and H. Hellwig (1977), Twofrequency excitation for the Ramsey separated oscillatory field method, J. Appl. Phys., 48, 5336-5337.

Klemperer, W. K. (1972), Long-baseline radio interferometry with independent frequency standards, Proc. IEEE, 60, 602609.

Kleppner, D., H. C. Berg, S. B. Crampton, N. F. Ramsey, R. F. C. Vessot, H. E. Peters, and J. Vanier (1965), Hydrogen maser principles and techniques, Phys. Rev., 138, A972-A983.

Kleppner, D., R. F. C. Vessot, and N. F. Ramsey (1970), An orbiting clock experiment to determine the gravitational red shift, A strophys. Space Sci, 6, 13-32.

Kusters, J. A., C. A. Adams, and H. Yoshida (1977), TTC'sfurther developmental results, Proceedings of the 31st Annual Frequency Control Symposium, pp. 3-7, Electronic Industries Association, Washington, D.C.

Lethokov, V. S., V. G. Minogin, and B. D. Pavlik (1977), Cooling and capture of atoms and molecules by a resonant light field, Eksp. Theor. Phis, 72, 1328-1341.

Levine, M. W., R. F. C. Vessot, and E. M. Mattison (1978), Performance evaluation of the SAO VLG-11 atomic hydrogen masers, Proceedings of the 32nd Annual Frequency Control Symposium, pp. 477-485, Electronic Industries Association, Washington, D.C

Mattison, E. M., M. W. Levine, and R. F. C. Vessot (1976), New TE ${ }_{11}$-mode hydrogen maser, Proceedings of the 8 th Precise Time and Time Interval Planning Meeting, pp. 355-368, Technical Information and Administrative Support Division, Goddard Space Flight Center, Greenbelt, Md.

McCaskill, T., J. White, S. Stebbins, and J. Buisson (1978), NTS-2 cesium frequency stability results, Proceedings of the 32nd Annual Frequency Control Symposium, pp. 560-566, Electronic Industries Association, Washington, D.C.

McCoubrey, A. O. (1966), A survey of atomic frequency standards, Proc. IEEE, 54, 116-135.

Missout, G., and J. Vanier (1975a), Pressure and temperature coefficients of the more commonly used buffer gases in rubidium vapor frequency standards, IEEE Trans. Instrum. Meas., IM-24, 180-184.

Missout, G., and J. Vanier (1975b), Some aspects of the theory of passive rubidium frequency standards, Can. J. Phys., 53, 1030-1043.

Mungall, A. G. (1978), A new concept in atomic time keeping: The continuously operating long beam primary cesium clock, IEEE Trans. Instrum. Meas., IM-27, 330.

Percival, D. B. (1976), A heuristic model of long-term atomic clock behavior, Proceedings of the 30th Annual Frequency Control Symposium, pp. 414-419, Electronic Industries Association, Washington, D.C.

Peters, H. E. (1978), Small, very small and extremely small hydrogen masers, Proceedings of the 32nd Annual Frequency Control Symposium, pp. 469-476, Electronic Industries Association, Washington, D.C.

Picque, J. L. (1977), Hyperfine optical pumping of a cesium atomic beam, and applications, Metrologia, 13, 115-120.

Reinhardt, V. S., H. E. Peters, and L. A. Birnbaum (1976), Field operable hydrogen maser design, Proceedings of the 8th Precise Time and Time Interval Planning Meeting, pp. 
197-212, Technical Information and Administrative Support Division, Goddard Space Flight Center, Greenbelt, Md.

Reisse, R. A. (1976), The effects of gravitational potential on atomic clocks as observed with the laser pulse time transfer system, Ph.D. thesis, Univ. of Md., College Park.

Ringer, D. E., J. Gandy, and E. Jechart (1975), Spaceborne rubidium frequency standard for NAVST AR GPS, Proceedings of the 7th Annual Precise Time and Time Interval Planning Meeting, pp. 671-677, Technical Information and Administrative Support Division, Goddard Space Flight Center, Greenbelt, Md.

Risley, A. S., and G. Busca (1978), Inhomogenous broadening and its relation to frequency instability in passive $\mathbf{R b}^{87}$ frequency standards, Proceedings of the 32nd Annual Frequency Control Symposium, pp. 506-513, Electronic Industries Association, Washington, D.C.

Rogers, A. E. E., A. R. Whitney, and L. B. Hanson (1976), A comparison of various hydrogen-maser frequency standards, Proceedings of the 8th Precise Time and Time Interval Planning Meeting, pp. 399-405, Technical Information and Administrative Support Division, Goddard Space Flight Center, Greenbelt, Md.

Rutman, J. (1977), Oscillator specifications: A review of classical and new ideas, Proceedings of the 31st Annual Frequency Control Symposium, pp. 291-301, Electronic Industries Asso. ciation, Washington, D.C.

Sabisky, E. S., and H. A. Weakliem (1978), An operating development model spacecraft hydrogen maser, Proceedings of the 32nd Annual Frequency Control Symposium, pp. 499505, Electronic Industries Association, Washington, D.C.

Séances de la 13 e Conference General des Poids et Mesures, October 1967 (1968), p. 103, Gauthier-Villars, Paris, France.

Smagin, A. G. (1974), A 1-MHz quartz resonator with a $Q$-factor of $4.2 \cdot 10^{9}$ at a temperature of $2^{\circ} \mathrm{K}$, Prib. Tekh. Eksp., $6,143-145$.

Smith, H. M. (1972), International time and frequency coordination, Proc. IEEE, 60, 479-487.

Stein, S. R. (1975), Application of superconductivity to precision oscillators, Proceedings of the 29th Annual Frequency Control Symposium, pp. 321-327, Electronic Industries Association, Washington, D.C.

Stein, S. R., C. M. Manney, F. L. Walls, and J. E. Gray (1978), A systems approach to high performance oscillators, Proceed- ings of the 32nd Annual Frequency Control Symposium, pp. 527-530, Electronic Industries Association, Washington, D.C.

Vanier, J., and R. Larouche (1978), A comparison of the wallshift of TFE and FEP teflon coatings in the hydrogen maser, Metrologia, 14, 31-38.

Vessot, R. F. C., and M. W. Levine (1977), A test of the equivalence principle using spaceborne clocks: General relativity and gravitation, Atti. Accad. Naz. Lincei, 34, 371-391.

Vessot, R. F. C., M. W. Levine, and E. M. Mattison (1977), Comparison of theoretical and observed maser stability limitation due to thermal noise and the prospect of improvement by low temperature operation, Proceedings of the 9 th Precise Time and Time Interval Planning Meeting, pp. 549-570, Technical Information and Administrative Support Division, Goddard Space Flight Center, Greenbelt, Md.

Walls, F. L., and D. A. Howe (1978), A passive hydrogen maser frequency standard, Proceedings of the 32nd Annual Frequency Control Symposium, pp. 492-498, Electronic Industries Association, Washington, D.C.

Wang, H. T. M., A. E. Popa, W. B. Bridges, and D. Schnelker (1977), Operational characteristics of a prototype spaceborne hydrogen maser, Proceedings of the 9th Precise Time and Time Interval Planning Meeting, pp. 415-424, Technical Information and Administrative Support Division, Goddard Space Flight Center, Greenbelt, Md.

Williams, R. E. (1976), A direct measurement of the relativistic effect of the gravitational potential on the rates of atomic clocks flown in aircraft, Ph.D. thesis, Univ. of Md., College Park.

Wineland, D. J. (1977), The cesium beam frequency standardprospects for the future, Metrologia, 13, 121-123.

Wineland, D. J., D. W. Allan, D. J. Glaze, H. Hellwig, and S. Jarvis, Jr. (1976), Results on limitations in primary cesium standard operation, IEEE Trans. Instrum. Meas., IM-25, 453-458.

Wineland, D. J., D. A. Howe, and M. B. Mohler (1977), Results with the special-purpose ammonia frequency standard, Proceedings of the 31st Annual Frequency Control Symposium, pp. 562-573, Electronic Industries Association, Washington, D.C.

Wineland, D. J., R. E. Drullinger, and F. L. Walls (1978), Radiation-pressure cooling of bound resonant absorbers, Phys. Rev. Lett., 40, 1639-1642. 